\title{
Media Stickiness and Cognitive Imprinting: Inertia and Creativity in Cooperative Work \& Learning with ICTs
}

\author{
Ruth Geer \& Alan Barnes \\ 1 Cognitive Technologies in Education Group \\ School of Education, Magill Campus \\ 2 University of South Australia \\ GPO Box 2471 Adelaide \\ Email: ruth.geer@unisa.edu.au \& alan.barnes@unisa.edu.au
}

\begin{abstract}
This paper attempts to build a bridge between the fields of Computer Supported Cooperative Work and learning in online communities. Of particular importance is their use of information and communication technologies. Each field has independently developed notions of inertia in the behaviour of users of these technologies. The notion of media stickiness is examined and related to that of imprinting in learning communities. Various suggestions are made of value to both fields and further research identified.
\end{abstract}

\section{Introduction}

The field of Computer Supported Cooperative Work (CSCW) has much to offer our understanding of the processes and possibilities for learning in communities using information and communication technologies (ICTs). While the traditional focus of distributed group work research has been in understanding how teams can work together and how to design better tools for them to do so, the tendency to focus on the outcomes and organisational goals rather than learning and conceptual goals has constrained the cross fertilisation of these fields.

This paper identifies one such area of fertile common ground and attempts to articulate the linkages and implications for understanding in both fields. In CSCW technology appropriation examines the basis for technology choice and adoption in virtual teams. Recently the concept of media stickiness [1] has emerged to account for the tendency of groups to stay with their early choice of ICTs. While in the study of learning in online communities the concept of cognitive imprinting [2] has emerged to account for the tendency for online learning groups to repeat early patterns of cognition. 
This study first outlines the notion of media stickiness in CSCW leading to considerations of cognitive development in learning. Secondly the discussion moves to an examination of interactive behaviours and resultant cognitive indicators through an analysis of the discourse from digital archives. Commonalities across the two fields of study are explored and directions for future research advanced.

\section{Media Stickiness}

Many industries are now global, some have manufacturing sections in one country, research and development, information technology and finance sections in others. With increasing fierce global competition and globalised workforces the need for effective methods for distributed cooperative work have never been greater and are bound to grow further in the $21^{\text {st }}$ century. The field of Computer Supported Cooperative Work (CSCW) has developed to underpin best practices through examining effective interactions in teams and develop better understandings of the design of effective computer tools and of their use. While the advantages of using ICTs to support distributed work groups include speed of communication, flexibility and adaptiveness, the disadvantages include lack of trust, coordination problems and lack of shared background knowledge among others [1]. In a study of six engineering design teams, Huysman et al. [1] show the evolution of communication technology use through a protracted joint design project. The teams were made up of students from Michigan State University (USA) and Deft University of Technology (Netherlands) who were forced by distance to communicate by online means. The technologies included desktop video conferencing, email, chat, shared white boards, real-time integrated audio and video as well as application sharing, a shared file system, calendar and message board. In this qualitative study, the students were allowed to choose the type of tools with which they wanted to work.

The six teams exhibited distinctly different patterns and preferences for these ICTs. Moreover such choices were made early in the design project and remained the preferred practice throughout the design process. This habitual inertia in the use of ICTs was evident in one group who when shown how useful the shared whiteboard could be, returned to their original ICT choices directly thereafter. The authors termed this effect 'media stickiness' because the initial choice of technology was retained despite more useful technologies being available. The study does not quantify the extent of this effect but demonstrates it in the evolution of discourse and practice within the project teams.

A number of explanations are given for this by the authors. One is the lack of feedback about communication processes both internal and external to the team. Another is the deadline for completion that induced a lack of interest in experimenting with styles of communication. Still another is their lack of familiarity and comfort in criticizing one another and expressing their feelings about problems with the team's communication. Finally, the authors considered that gaining mutual agreement for such a change was simply too much effort.

The style of media use becomes so entrenched in the culture of teams that after a while it appears impossible to change it. Tyre \& Orlikowski [3] suggest that after 
initial modification to groupware, the windows of opportunity for users to change the way the tools are used and configured is limited. This suggests path dependency in groupware use.

The media stickiness study is important for the better understanding of online learning communities and the role of student choice. After all, students in the $21 \mathrm{st}$ century will have a wide range of ICTs at their disposal. Hence should educators try to constrain this choice or find ways to influence good choice? This will become increasingly relevant both to formal online learning community design and informal but important communications between students.

\section{Cognitive development in learning}

There is still much to learn about the impact of computer technologies on human actions and cognitive performance. Research into the interactive behaviour and cognitive development in online learning communities is constantly seeking increased understanding. One study using asynchronous online discussion forums identified a number of findings which help to explain the cognitive behaviour of learners. Education for the $21^{\text {st }}$ century requires an understanding of cognition and its relation to the technologies.

Interaction and collaboration are recognized as key ingredients in such environments [4]. However it cannot be assumed that learners automatically know how to interact and collaborate to achieve the desired learning outcomes. Educators must carefully build these experiences into the educational design. Later researchers [5-8] have added to these critical elements with a body of knowledge emerging that identifies additional elements and addresses how individuals learn with the technologies. With constantly emerging technologies educators face the predicament of which technologies are best suited to support the required learning outcomes and how to utilize these technologies which are evolving more rapidly than the pedagogies.

Due to a lack of pedagogical guidance about integrating technologies for collaboration and communication, educators are left with mounting dilemmas and confusion [9]. Currently, the corporate world drives much of the information economy and influences the development of information technologies. Educators require a greater say in the evolution of technologies that will equip students with the cognitive skills that make them successful lifelong learners. Technologies that support greater collaborative activities are emerging slowly, as educators push for more collaborative interactivity. One source for these technologies should be the field of CSCW.

\section{Imprinting}

Initial communication patterns have been shown to be powerful in determining subsequent interactive behaviours in the learning communities. In a recent study [2] an analysis of the impact of these initial communications has led to the notion of 
"imprinting" as a means of characterising the scrially consistent cognitive behaviour of the students within discussion forums. The effects of imprinting then become a consideration in the formation of discussion forums or online learning communities. From a teaching and learning perspective this implies that the cognitive behaviours that occur in the early interactions therefore need to reflect the desired learning outcomes, if the discussion forums are to shift students cognitively.

In this study, the discourse analysis of 15 discussion forums ( 275 students) over 4 years and across 4 topics in a first year teacher education course showed consistency in communication patterns across the various topics in each discussion forum. These forums with an approximate size of 20 students provided an opportunity for students to research and discuss authentic and relevant topics relating to the use of technologies in the classroom. An example of the topics was "In relation to the research literate what are the major issues surrounding the use of the word processor in the junior primary classroom?" Within each forum students were divided into four smaller groups each of which then facilitated the discussion of one of the topics and presented a summary of the main issues in a face to face presentation. The students were required to respond with at least one 300-400 word contribution for each topic with topics posted at regular intervals throughout the semester.

An adaptation of Gunawardena, Lowe and Anderson's, Interaction Analysis Model [10] for examining social construction of knowledge in computer conferencing, provided a basis for developing an effective evaluative model for analysing archived discourse. Adaptations to this model drew on Garrison, Anderson and Archer's Community of Inquiry model [11] and Henri's five dimensions of learning [12]. This evaluative model (Table 1) supported an investigation of the discourse at three levels. The three types of learner orientations (social, individual and group) identified the interactive behaviour of the participants. The phases within each orientation characterised the cognitive activity, while the indicators within the phases showed the approaches to learning that were being adopted.

Table 1. A model for Social Behaviour, Cognitive Development and Interactive Analysis

\section{S. Participation and social behaviour}

S1 Individual disclosure

S1-a Basic introduction.

S1-b Extended revelation

S1-c Self evaluation

S2 Social behaviour

S2-a Courtesy

S2-b Level of dominance/authority

S2-c Seeking help

S2-d Willingness to initiate

S3 Mutual Consideration

S3-a Identifying mutual interest

S3-b Willingness to exchange

S3-c Valuing others' views 


\section{Cognitive behaviour analysis at individual level:}

I1 Elementary clarification

I1-a Observing/studying a problem

I1-b Identifying its elements

Il-c Observing/studying their linkages

I2 In-depth clarification

I2-a Analysing a problem

I2-b Identifying assumptions

I2-c Establishing referential criteria

I2-d Seeking out specialized information

I3 Synthesis and application

I3-a Drawing primary conclusions

I3-b Proposing an idea based on links and relevant information

I3-c Value judgment on relevant solutions

I3-d Making final decisions and deciding on the action(s) to be taken

\section{G. Interactive behaviour analysis at group level:}

G1 Planning

G1-a Organizing work/planning group work/setting shared tasks

G1-b Initiating activities/setting up activities for group work

G2 Sharing/comparing/contributing of information

G2-a Defining and identifying a problem

G2-b Stating opinions regarding the problem

G2-c Asking and answering questions to clarify details of statements

G2-d Sharing and exchanging knowledge, resources and information

G2-e Corroborating examples provided by one or more participants

G2-f Challenging others to engage in group discussion

G2-g Help and feedback giving

G3 Inconsistency of ideas, concepts or statements

G3-a Identifying and stating areas of disagreement

G3-b Asking and answering questions to clarify the source and extent of disagreement

G3-c Restating the participants' position and advancing arguments or considerations supported by references

G4 Negotiation of meaning/co-construction of knowledge

G4-a Negotiating or clarifying the meaning of terms, areas of agreement and disagreement

G4-b Proposing new statements embodying compromise and co-construction

G4-c Integrating or accommodating metaphors or analogies

G5 Testing and modification of proposed synthesis or co-construction of knowledge

G5-a Testing against existing knowledge and information

G5-b Testing against personal experience

G5-c Testing against formal data collected

G6 Agreement statement(s) and application of newly constructed knowledge

G6-a Summarization of agreement(s)

G6-b Application of new knowledge 
Using the evaluative model cognitive indicators were recorded for each topic as well as their aggregates for each student against social (S), individual (I) and group (G) orientations. The data as illustrated in the figures below showed a strong level of continuity across the 4 topics in the individual and group aggregates and a weaker but still positive level of continuity in the case of social aggregates across topics based on the indicators from the evaluative model.

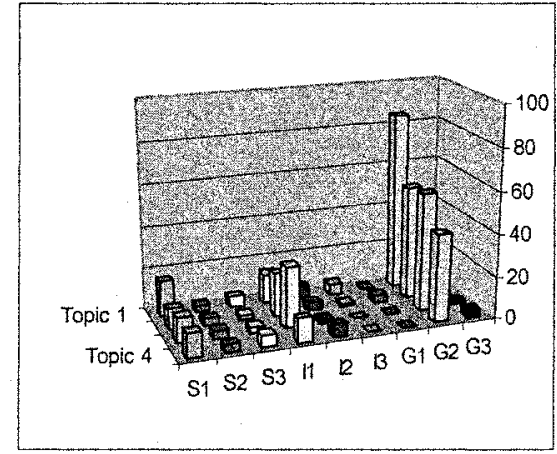

Figure 1. Group oriented forum

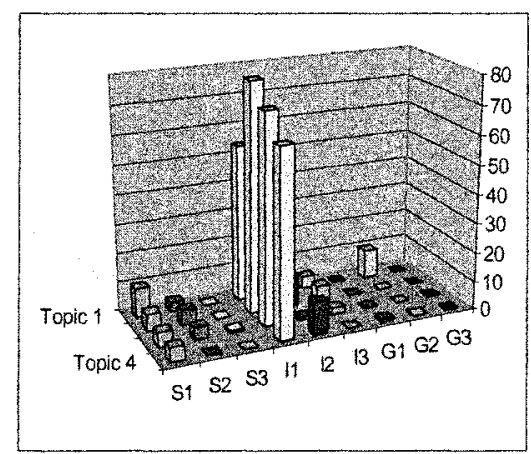

Figure 2. Individually oriented forum

Such analysis provided insight into the cognitive activity of the participants and identified two types of imprinting that were occurring over time, see Figure 3 . In some instances one particular indicator may dominate the discussion (such as elementary clarification, I1 or sharing/comparing information, G2) which prevails across the discussion topics (Type A). In these cases the cognitive development remains static across the topics. 'Static' describes the process where there is little or no change in indicators over a period of time. Furthermore, students may demonstrate a set of cognitive indicators for each topic that are repeated for subsequent topics (Type B). Here students show an appreciation of differing cognitive strategies and knowledge acquisition for each topic. Their responses for each topic may demonstrate a cognitive movement through the indicators; such as starting with problem identification which progresses to analysis and the drawing of some conclusions. However, rather than furthering the development of learning approaches, students use the same set of indicators for each topic indicating a broader but static track.

It is helpful to consider the record of cognitive indicators in the course as a cognitive track which may be relatively straight and narrow as students focus on a particular learning approach, such as I1a, I2a or G2a, wavering very little in their approach to each topic (Figure 3). Alternatively it may be a broader track as students explore, investigate and interpret different approaches to knowledge acquisition. These two types of imprinting will be referred to as 'static cognitive tracks' where the cognitive engagement is consistent across the topics. Hence if the aim of the discussion forum is to have students analyse a topic showing cognition phases of say, I2 or G2, then imprinting will ensure that narrow track, Type A, occurs across the topics. However if the intention of the forums is to develop students' higher order thinking then an example of a broad track, Type B, should be evident. This may 
have implications on how technologies are used by the forums in their discussion. Such static tracks may be a goal of the educator.

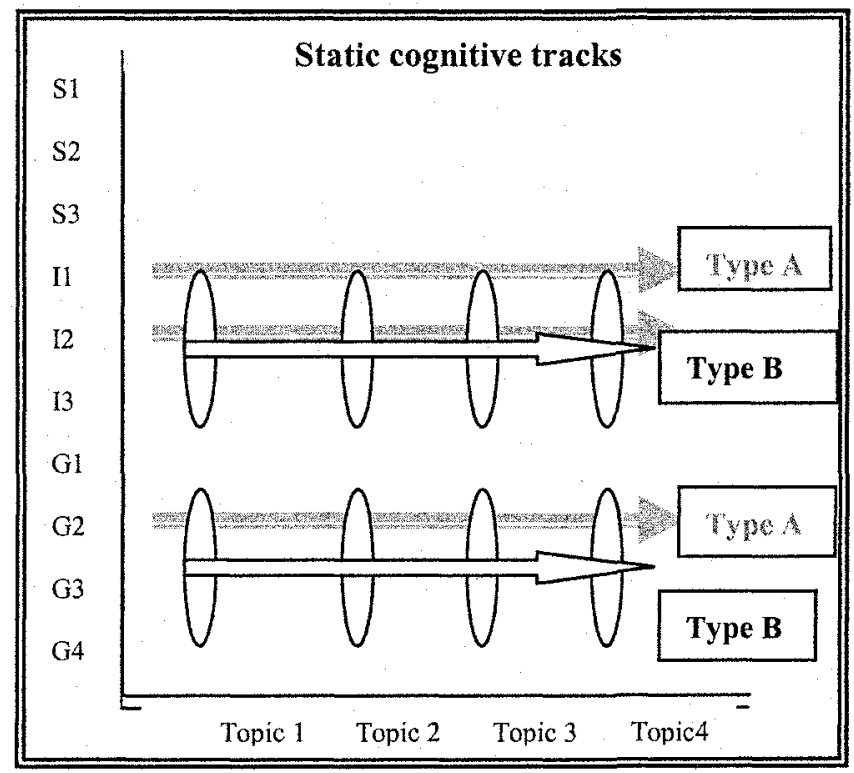

Figure 3. Imprinting of cognition across time

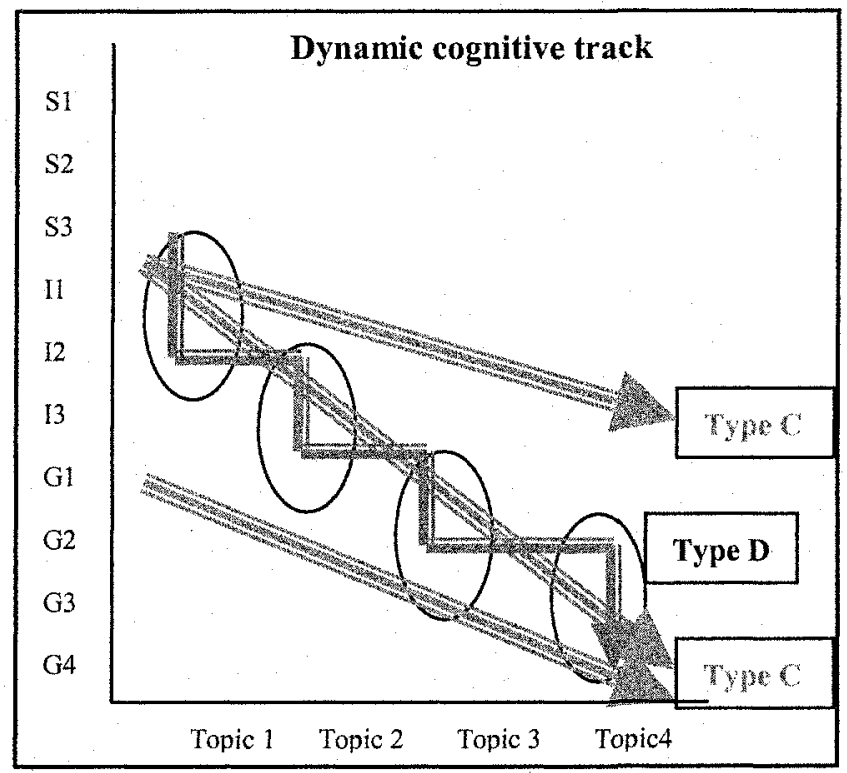

Figure 4. Progressive cognitive development through time 
On the other hand, if educators wish to avoid imprinting because they desire students to move progressively rather than statically along various cognitive tracks, then factors that lead to imprinting must be counterbalanced.

The term 'dynamic cognitive track' suggests a change in the cognitive focus where students move through various indicators (Table 1) and perhaps to higher levels of cooperation or collaboration. Again in Figure 4 there are narrow and broad cognitive tracks corresponding to the breadth of cognitive indicators displayed at any one time by the learner.

\section{Conclusion}

The media stickiness study examined patterns of media use by engineering students as they communicated about a design project with multiple ICT options. The cognitive imprinting study established patterns of cognition in students communicating their understanding about specific topics with a single ICT (email discussion lists). Both demonstrate that the initial patterns can become established and difficult to shift. The imprinting study with its in depth examination of the cognitive concomitants of the discourse is able to reach a deeper level of analysis. Indeed it is legitimate to argue that if a group of indicators similar to those in Table 1 above had been applied to the discourse of the media stickiness study, it would have exposed the cognitive basis of the patterns of media use. The various explanations offered by the authors could then be put to the test. Such an argument would see inertia in both studies as being cognitive in its origins. Media stickiness then emerges as an aspect of cognitive inertia.

An examination of the limited excerpts from student in the media stickiness study suggests that there is a link been social relationships (S1-S3) and learner comfort in being able to express their criticisms. The movement over time between exhibiting simple problem solving to that of testing assumptions (I2a-I2b) would be relevant to such virtual teams and help uncover discrepancies in background knowledge and goals. It is notable that the Michigan students considered that the project was focused on building workable systems in practice while the Delft students were more interested in conceptual issues and model testing (G3a-G3b). It is important to ask whether the cognitive behaviour in the media stickiness study, and in virtual teams in general, is predominantly individual or of group orientation. The later more easily leads to creative cooperation than collaboration.

The use of a set of cognitive concomitants in CSCW might prove useful as an early warning system by giving indications that specific aspects of interactions are or are not occurring, just as it can in learning communities. Indeed both studies point to the focusing on the critical formative periods at the beginning of engagements where resources and interventions may have long term value. Use of such indicators can also help understand when the cognitive concomitants of creativity and original collaboration can be achieved.

This exchange between two disparate fields raises more questions than it answers. What are the best ways to subvert cognitive inertia and advance creative learning and groupwork? In what ways can dynamic cognitive tracks be quickly 
identified? What semi-automated processes, such as key phrase searches, could support identification of critical changes or lack of them in cognitive concomitants? Students in the 21 st century will have much more experience of multiple ICTs. How will personal preferences and habits for ICTs clash with those of the work group or learning community? Can such a model of cognitive concomitants, designed to analyse email discussion, be robust enough to deal with white boards and other visual media? Are there ways to distinguish between cooperative and collaborative interaction in visual media? Indeed the nature of the cognitive concomitants that can effectively characterise interaction in such media is a vital question for the respective futures of both fields.

\section{References}

1. M. Huysman, C. Steinfield, C. Y Jang, K. David, M. Huis in 'T Veld, J. Poot, et al., Virtual Teams and the Appropriation of Communication Technology: Exploring the Concept of Media Stickiness, Computer Supported Cooperative Work, 12, 411-436 (2003).

2. R.E. Geer, Interaction and Cognition in Online Learning Communities, Doctor of Philosophy (University of South Australia, Adelaide, 2005).

3. M.J. Tyre and W.J. Orlikowski, Windows of Opportunity: Temporal Patterns of Technological Adaptation in Organisations, Organization Science, 5(1), 98-1 18 (1994).

4. J.L. King \& D.L Doerfert, Interaction in the Distance Education Setting. (September 7, 1999); http://www.ssu.missouri.edu/ssu/Aged/NAERM/s-e-4.htm

5. S. Agostinho, R. Oliver, B. Harper, J. Hedberg and S. Wills, A Tool to Evaluate the Potential for an ICT-based Learning Design to Foster High Quality Learning. Paper presented at the 19th Annual Conference of ASCILITE, Auckland, NZ (2002).

6. D.R Garrison, T. Anderson and W. Archer, Critical Inquiry in a Text-based Environment: Computer Conferencing in Higher Education. The Internet \& Higher Education, 2(2-3), 1-19 (2000).

7. C. McLoughlin, Computer Supported Teamwork: An Integrative Approach to Evaluating Cooperative Learning in an Online Environment. Australian Journal of Educational Technology, 18(2), 227-254 (2002).

8. E. Stacey and M. Rice, Evaluating an Online Learning Environment. Australian Journal of Educational Technology, 18(3), 323-340 (2002).

9. C. J. Bonk and D.J. Cunningham, Searching for Learner-Centered, Constructivist and Sociocultural Components of Collaborative Educational Learning Tools. In Electronic Collaborators: Learner-Centred Technologies for Literacy Apprenticeship and Discourse edited by C.J. Bonk and K.S. King (Mahwah, New Jersey: Lawrence Erlbaum Associates, 1998) pp. 25-50.

10. C.N. Gunawardena, C. Lowe and T. Anderson, Analysis of a Global Online Debate and the Development of an Interaction Analysis Model for Examining Social Construction of Knowledge in Computer Conferencing. Journal of Educational Computing Research, 17(4), 397-431 (1997). 
11. D.R. Garrison, T. Anderson, and W. Archer, Critical Inquiry in a Text-based Environment: Computer Conferencing in Higher Education, The Internet \& Higher Education, 2(2-3), 1-19 (2000).

12. F. Henri, Computer conferencing and content analysis, in Collaborative learning through computer conferencing: The Najaden Papers, edited by A. Kaye, (Springer-Verlag: Berlin. 1992) pp. 117-136. 